\title{
Simulated evolution process of core-shell microstructures
}

\author{
QIN Tao, WANG HaiPeng \& WEI BingBo ${ }^{\dagger}$ \\ Department of Applied Physics, Northwestern Polytechnical University, Xi'an 710072, China
}

The evolution process of core-shell microstructures formed in monotectic alloys under the space environment condition was investigated by the numerical simulation method. In order to account for the effect of surface segregation on phase separation, Model $\mathrm{H}$ was modified by introducing a surface free energy term into the total free energy of alloy droplet. Three $\mathrm{Fe}-\mathrm{Cu}$ alloys were taken as simulated examples, which usually exhibit metastable phase separation in undercooled and microgravity states. It was revealed by the dynamic simulation process that the formation of core-shell microstructures depends mainly on surface segregation and Marangoni convection. The phase separation of $\mathrm{Fe}_{65} \mathrm{Cu}_{35}$ alloy starts from a dispersed structure and gradually evolves into a triple-layer core-shell microstructure. Similarly, $\mathrm{Fe}_{50} \mathrm{Cu}_{50}$ alloy experiences a structural evolution process of "bicontinuous phase $\rightarrow$ quadruple-layer core-shell $\rightarrow$ triple-layer core-shell", while the microstructures of $\mathrm{Fe}_{35} \mathrm{Cu}_{65}$ alloy transfer from the dispersed structure into the final double-layer core-shell morphology. The $\mathrm{Cu}$-rich phase always forms the outer layer because of surface segregation, whereas the internal microstructural evolution is controlled mainly by the Marangoni convection resulting from the temperature gradient.

space environment, Fe-Cu alloy, phase separation, core-shell structure, numerical simulation

Liquid phase separation of monotectic alloys has aroused great interest in the fields of materials physics and space science ${ }^{[1-3]}$. The earlier researches show that surface segregation and Marangoni convection resulting from the temperature gradient play an important role in the microstructural evolution under the space environment condition ${ }^{[2,4]}$. Due to the opacity of metals and the form requirements of the experiments, it is quite difficult to carry out theoretical investigation deeply. Therefore, the evolution process and the micro-mechanism are still poorly understood. Numerical simulation has become an important method to explore phase separation. However, it has been exclusively implemented to study the pattern formation of polymers and little attention has been

Received February 11, 2007; accepted May 29, 2007 doi: $10.1007 / \mathrm{s} 11433-007-0045-7$

†Corresponding author (email: bbwei@nwpu.edu.cn)

Supported by the National Natural Science Foundation of China (Grant Nos. 50121101 and 50395105) and the Doctorate Foundation of Northwestern Polytechnical University (Grant No. CX200420) 
paid to the metal systems ${ }^{[5,6]}$. Furthermore, the previous numerical studies are primarily focused on the phenomena in rectangular geometries at a fixed temperature, and little work deals with the processes in other geometries with nonuniform additional fields. Under the space environment condition, liquid droplets form perfect spheres with the temperature gradient inside ${ }^{[1,7]}$, therefore, the traditional simulation methods are inapplicable.

Model $\mathrm{H}$ is of great success in the simulation of phase separation ${ }^{[8]}$. It includes the coupling of the flow field and concentration field, and is frequently used to study the bulk phase separation. Its application in the phase separation with surface segregation is still limited.

$\mathrm{Fe}-\mathrm{Cu}$ peritectic alloy is one of the most important metastable monotectic systems. Its equilibrium phase diagram ${ }^{[9]}$ is shown in Figure 1. The matrix decomposes to Fe-rich and $\mathrm{Cu}$-rich phases when it is undercooled to the miscible zone over a broad composition range. Due to the thermodynamic metastability, the phase separation can easily be suspended by the growth of $\gamma \mathrm{Fe}$ dendrites, and produce various microstructures ${ }^{[7,8]}$. Although some researches have been carried out to explore its metastable phase separation behaviors, further efforts are still needed to reveal the microstructural evolution process and the mechanisms.

In this paper, numerical simulation is performed to investigate the microstructural evolutions of highly undercooled $\mathrm{Fe}-\mathrm{Cu}$ alloys droplets under the space environment condition and the effects of the surface segregation and temperature gradient are analyzed. Three selected compositions in the simulations are shown in Figure 1.

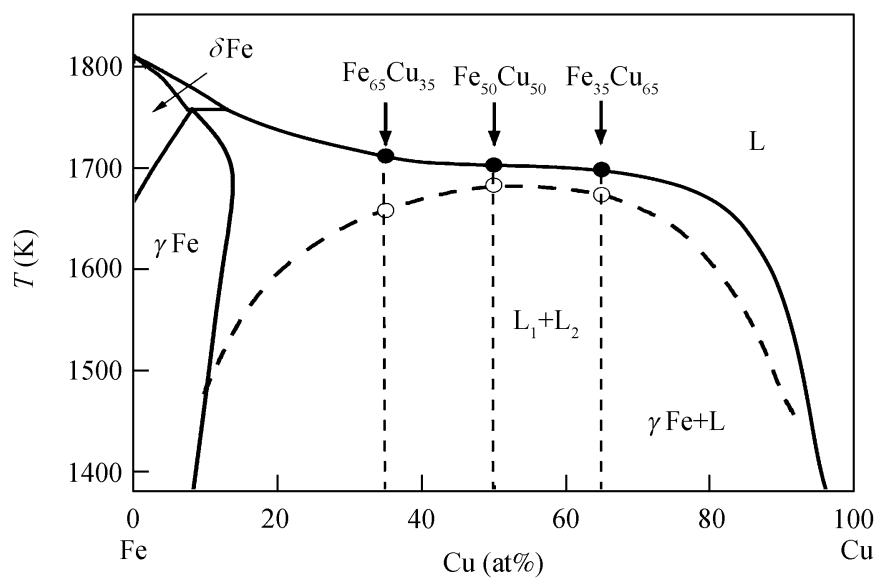

Figure 1 Three alloys selected from Fe-Cu binary system.

\section{Mathematical model and numerical method}

The liquid phase separation can be described by Model $\mathrm{H}$, given in the dimensionless form as ${ }^{[10,11]}$

$$
\frac{\partial \phi}{\partial \tau}=-\nabla \cdot(\boldsymbol{v} \phi)+\nabla \cdot\left[\phi(1-\phi) \nabla \frac{\delta F}{\delta \phi}\right]+\nabla \cdot \xi,
$$

where $\phi$ denotes the molar fraction of the segregating component, i.e. $\mathrm{Cu}$ in this work, $\tau$ is the time, $v$ the local velocity, $\delta$ the Lagrange variation operator, and $\xi$ the random Gaussian white noise with the fluctuation amplitude $\varepsilon$, and $F$ the free energy functional.

Previously, only the bulk free energy $F_{\mathrm{b}}$ and the contribution from the concentration gradient 
$F_{\text {grad }}$ were considered when describing the system free energy $F$. In fact, the surface free energy $F_{\mathrm{S}}$ also plays a crucial role in phase separation. In the presence of surface segregation, the surface free energy $F_{\mathrm{S}}$ has to be involved. Thus, $F$ should be written by

$$
F=F_{\mathrm{b}}+F_{\text {grad }}+F_{\mathrm{S}} .
$$

It is reasonable to take $F_{\mathrm{b}}$ to be one of the regular solutions, i.e.

$$
F_{\mathrm{b}}=F_{\mathrm{b} 0}+\theta[\phi \ln \phi+(1-\phi) \ln (1-\phi)]+2 \phi(1-\phi),
$$

where $F_{\mathrm{b} 0}$ is the constant part, $\theta$ the reduced temperature, defined as $T / T_{\mathrm{L}}$, and $T_{\mathrm{L}}$ the critical temperature. $F_{\text {grad }}$ is given as

$$
F_{\text {grad }}=\frac{1}{2} \nabla^{2} \phi
$$

$F_{\mathrm{S}}$ is taken as ${ }^{[12]}$

$$
F_{\mathrm{S}}=F_{\mathrm{S} 0}-H \phi_{\mathrm{S}}+\frac{1}{2} g \phi_{\mathrm{S}}^{2}
$$

where $\phi_{\mathrm{S}}$ represents the surface concentration, and $F_{\mathrm{S} 0}, H$ and $g$ are constants, describing the surface preferential attraction properties.

In small droplets, the Reynolds number is less than the magnitude of $10^{-3}$, therefore, the local velocity can be taken proportionally to the local body force $\boldsymbol{f}=-\nabla F^{[13]}$. Expending $\nabla F$ gives

$$
v=-\alpha \cdot \phi \nabla \frac{\delta F}{\delta \phi}
$$

with

$$
\alpha=\frac{\rho R_{\mathrm{g}} T_{\mathrm{L}} l^{2}}{6 \pi D_{\mathrm{L}} M \eta},
$$

where $\rho$ is the mass density, $l$ the interface length ${ }^{[14]}, R_{\mathrm{g}}$ the gas constant, $D_{\mathrm{L}}$ the diffusion coefficient, $M$ the molar weight, and $\eta$ the viscosity. $\alpha$ reflects the fluidity of the alloy melt, and the larger it is, the more severely the flow field responds to the local force field.

Eqs. (1) - (7) are supplemented with the following boundary conditions at the surface $R=R_{0}$,

$$
\begin{gathered}
\left.\boldsymbol{n} \cdot \nabla \phi\right|_{R_{0}}=0 \\
\left.\boldsymbol{n} \cdot \nabla \frac{\delta F}{\delta \phi}\right|_{R_{0}}=0,
\end{gathered}
$$

where $\boldsymbol{n}$ gives the normal direction at the surface. The incompressibility condition on the fluid $(\nabla \cdot v=0)$ is not applied here, and the justification is that this effect is not significant as numerically validated by Chen and Chakrabarti ${ }^{[15]}$.

To incorporate the influence of the temperature gradient on the phase separation, a nonuniform temperature field is applied. It decreases linearly along the radial distance from the center to the surface with the total temperature difference of $5 \mathrm{~K}$. The temperature at the surface is set to be $0.8 T_{\mathrm{L}}$, corresponding to a highly undercooled state.

The initial concentration is set at $\phi_{0}(=0.35,0.50$ and 0.65$)$ with random fluctuations of amplitude 0.05 . The initial local velocity is zero. The concentration fluctuation amplitude $\varepsilon$ in the simulation is 0.01 and no fluctuation of the velocity field is considered. The surface parameters are $H=0.35$ and $g=0.4$. $\varepsilon, H$ and $g$ are determined empirically. $\alpha$ is estimated as 1000 for the alloys.

The above equations are solved in two-dimensional Cartesian coordinates using an explicit finite 
difference scheme. The grid spacings are $\Delta x=\Delta y=1$ with a fixed lattice size $R_{0}=100$, and the time step is $\Delta \tau=0.0001$ to ensure the stability. The calculations were performed in a Lenovo 1800 cluster system.

\section{Results and discussion}

\subsection{Phase separation of $\mathrm{Fe}_{65} \mathrm{Cu}_{35}$ alloy}

The microstructural evolution snapshots from the simulation of $\mathrm{Fe}_{65} \mathrm{Cu}_{35}$ at different times are shown in Figure 2. Obviously, in the early stage, surface segregation occurs prior to the bulk decomposition, and leads to the formation of a $\mathrm{Cu}$-rich layer followed by a Fe-rich layer at the surface ( $\tau=0.1$ ). In the bulk, as the minor phase, the Cu-rich spheres form and grow diffusively $(\tau=5)$. After $\tau=5$, the hydrodynamic effect begins to behave visibly. The flow field quickly responses to the local force field and the coalition becomes much faster, and the $\mathrm{Cu}$-rich spheres grow rapidly $(\tau=50)$. Since the thermo-capillary effect is inherently included in the numerical model, the resultant Marangoni motion of the $\mathrm{Cu}$-rich spheres towards the hotter, i.e. the central area, can be explicitly observed in the simulation $(\tau=50,100,150$ and 260). The spheres aggregate at the central part and the triple-layer core-shell structure forms in the end.

In order to explore the influences of surface segregation and Marangoni convection on the concentration field during phase separation, the radial $\mathrm{Cu}$ distributions at different times were computed, as presented in Figure 3. The angular $\mathrm{Cu}$ concentration $\phi_{\text {av }}$ denotes the average value over the range of $[r-0.5, r+0.5]$ and every point set is averaged over 10 independent runs. According to Figure 3, due to the surface enrichment of $\mathrm{Cu}$, the concentration profile forms a crest and a trough near the surface in the beginning $(\tau=0.1)$. As the phase separation continues, in the Fe-rich layer, more $\mathrm{Cu}$ is exuded into the two lateral sides and more $\mathrm{Fe}$ is absorbed from them. Consequently, new layers rich in $\mathrm{Cu}$ and $\mathrm{Fe}$ form at the inner side. Therefore, the concentration profiles are shaped as waves by this chain reaction $(\tau=5)$. The $\mathrm{Cu}$-rich phases gradually coarsen and move towards the center area, and consequently, the waves propagate deeper into the bulk ( $\tau=$ $150)$.
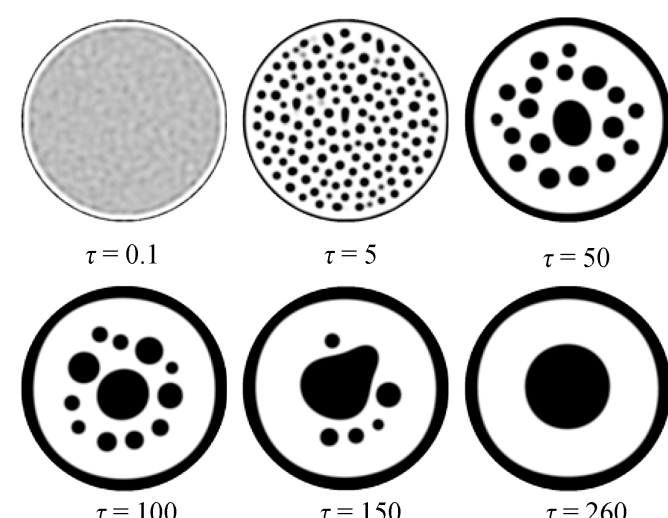

$\tau=100$

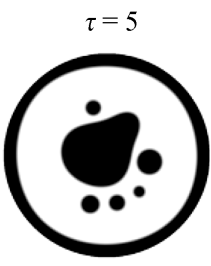

$\tau=150$

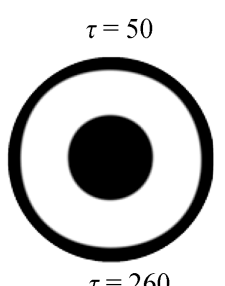

$\tau=260$

Figure 2 Snapshots from microstructural evolution process of $\mathrm{Fe}_{65} \mathrm{Cu}_{35}$ alloy. The grey level varies linearly between black and white, corresponding to the equilibrium concentrations $\phi_{\mathrm{e} 1} \approx 0.86$ and $\phi_{\mathrm{e} 2} \approx 0.15$, respectively.

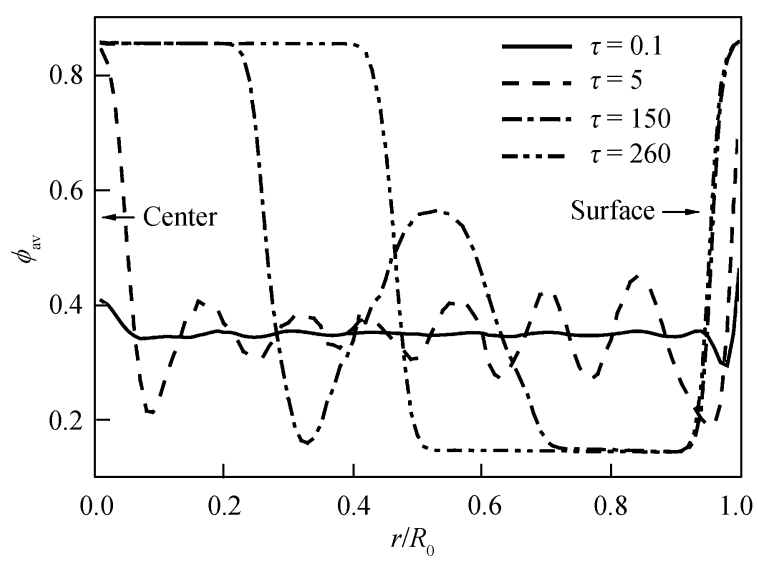

Figure $3 \mathrm{Cu}$ concentration $\phi_{\mathrm{av}}$ versus radial distance. 
Based on the above investigation on the concentration distribution, the thickness and the growth rate of the surface $\mathrm{Cu}$-rich layer were determined to further study the growth behaviors of the surface layer. The results are exhibited in Figure 4. It can be seen that the surface layer grows rapidly at first, gradually slows down and reaches a plateau. This deceleration can be explained by the competition between the surface segregation of $\mathrm{Cu}$ atoms and the Marangoni convection of the $\mathrm{Cu}$-rich spheres. In the beginning, it is very easy for $\mathrm{Cu}$ to diffuse to the surface, and the growth speed is quite large. As the phase separation progresses, the $\mathrm{Cu}$-rich spheres gradually move to the central part, less and less $\mathrm{Cu}$ diffuses through the Fe-rich layer to the surface layer, and consequently, the thickening slows down.

In order to understand the thermodynamics at different stages of phase separation, the evolutions of the total and partial free energies were also calculated in the simulation, as illustrated in Figure 5. The constant parts $F_{\mathrm{b}}$ and $F_{\mathrm{S}}$ are set to be zero when computed according to eqs. (3) and (4), respectively. All the data sets are averaged over 10 independent runs. It can be seen that the total free energy $F$ decreases monotonously with the time and the surface energy $F_{\mathrm{S}}$ quickly drops to the minimum value and keeps at a rough constant. The bulk free energy $F_{\mathrm{b}}$ and the gradient energy $F_{\text {grad }}$ evolve differently. At the early time, the matrix decomposes and interfaces quickly form. Consequently, $F_{\mathrm{b}}$ decreases and $F_{\text {grad }}$ increases steeply. During this time, the structural evolution is dictated by $F_{\mathrm{b}}$. When $\tau$ exceeds $2, F_{\text {grad }}$ begins to drop, and the reduction of the interfacial energy becomes dominant. This effect is embodied in two aspects: one is the coarsening of the phase domains to reduce the interfacial area; the other is the Marangoni motion towards the hotter area to minish the interfacial energy intensity.

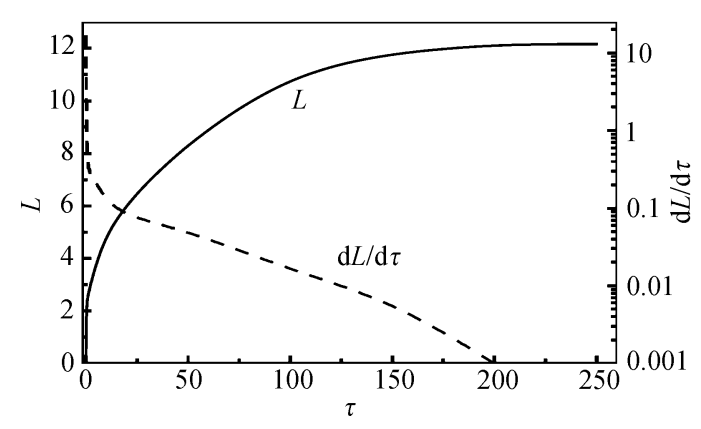

Figure 4 Surface layer versus dimensionless time.

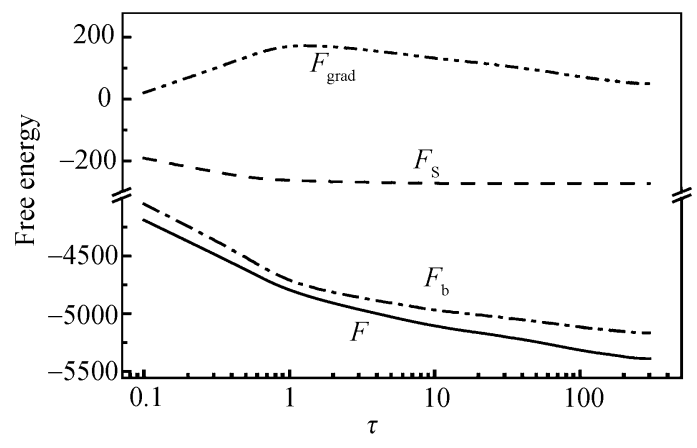

Figure 5 Variation of free energy with dimensionless time.

\subsection{Phase separation of $\mathrm{Fe}_{50} \mathrm{Cu}_{50}$ alloy}

The microstructural evolution process of $\mathrm{Fe}_{50} \mathrm{Cu}_{50}$ alloy is illustrated in Figure 6, and the corresponding concentration profiles are shown in Figure 7. At this composition, the surface segregation process is similar to that of $\mathrm{Fe}_{65} \mathrm{Cu}_{35}$. The surface layer is formed and it thickens quite fast initially and then grows slower and slower to the maximum value. Inside the droplet, the matrix separates to bicontinual phases instead of spheres, due to the same amount of the two components. After the hydrodynamic effects induced by the interfacial energy begin to dominate, the microstructure evolves from bicontinual phases to a quadruple-layer core-shell, and to a triple-layer core-shell eventually. The growth behavior of the surface layer and the free energy variation are similar to those of $\mathrm{Fe}_{65} \mathrm{Cu}_{35}$ alloy as shown in Figures 4 and 5. 


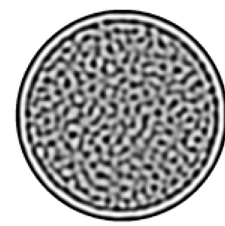

$\tau=0.1$

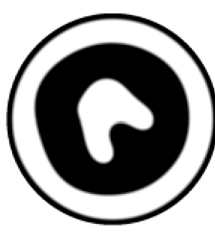

$\tau=30$
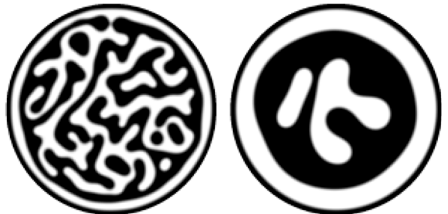

$\tau=20$

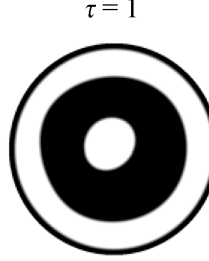

$\tau=50$

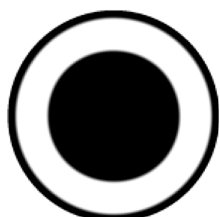

$\tau=200$

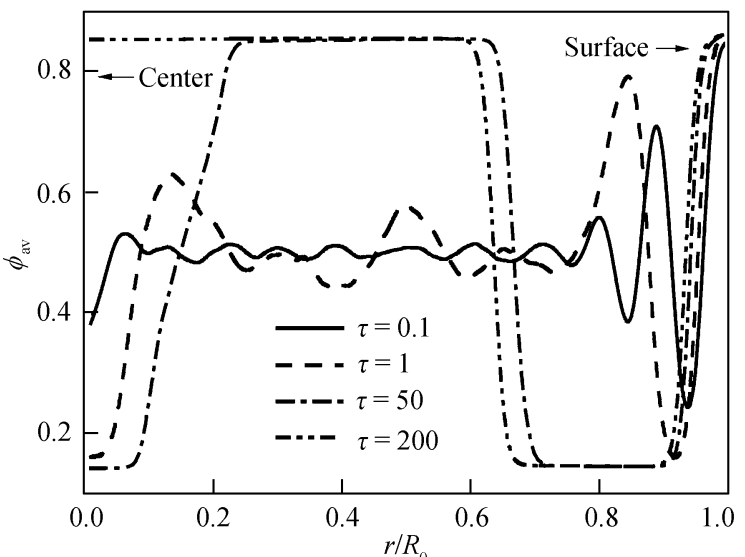

Figure $7 \mathrm{Cu}$ concentration versus radial distance for $\mathrm{Fe}_{50} \mathrm{Cu}_{50}$ alloy. alloy.

\subsection{Phase separation of $\mathrm{Fe}_{35} \mathrm{Cu}_{65}$ alloy}

Figure 8 gives the microstructural evolution of $\mathrm{Fe}_{35} \mathrm{Cu}_{65}$ alloy, and the corresponding concentration profile variations are plotted in Figure 9. Since the segregating component is the majority, no Fe-rich layer forms near the surface, whereas the concentration fluctuation similar to those in the former alloys is still aroused, as can be seen from Figure 9. The minor Fe-rich phases turn out in spheres, and grow through diffusion at the early stage and coagulate by Marangoni convection at later time. The microstructure evolves from a dispersed morphology to a double-layer core-shell.

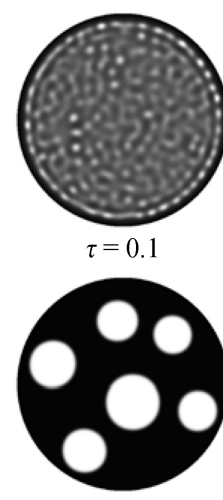

$\tau=50$

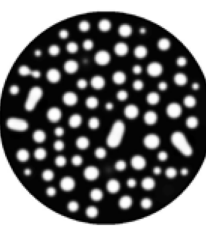

$\tau=1$

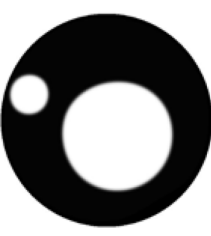

$\tau=250$

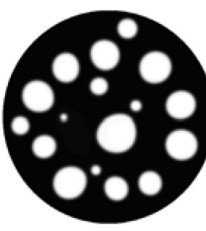

$\tau=10$

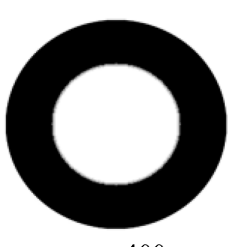

$\tau=400$

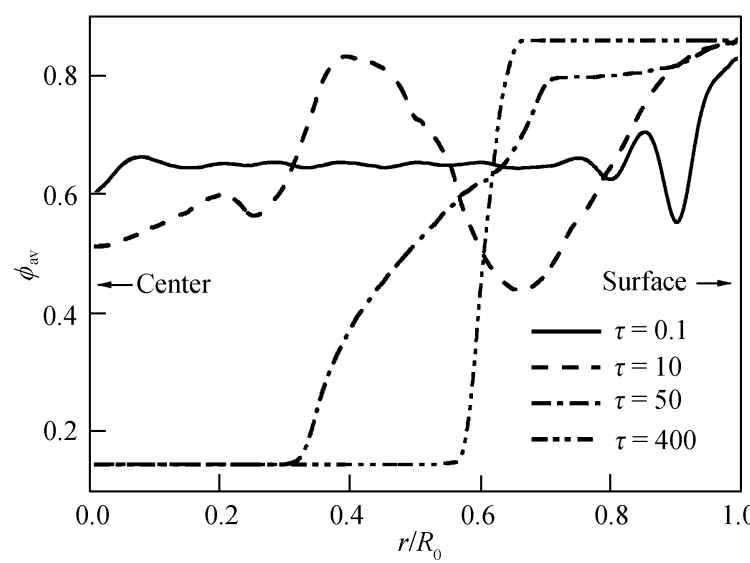

Figure $9 \mathrm{Cu}$ concentration versus radial distance for $\mathrm{Fe}_{35} \mathrm{Cu}_{65}$ alloy. alloy.

Finally, it is mentioned that the present approach can be extended to deal with the phase separation during rapid solidification by adding the effect of the temperature evolution, and the simulations of other immiscible alloys can also be applied by choosing proper free energy functionals and parameters. Owing to lack of experimental results describing the time evolution of the microstructures of those alloys, a comparison of the present simulations with other investigations is limited. It is expected that the above results will provoke fresh experiments on these problems. 


\section{Conclusions}

(1) Model $\mathrm{H}$ has been modified by taking into account the effect of surface free energy so that it becomes applicable to the liquid phase separation process under the influences of surface segregation. As a practical application, the evolution process of core-shell microstructures of undercooled $\mathrm{Fe}-\mathrm{Cu}$ alloys in space environment was numerically simulated in the light of this modified Model H.

(2) According to the simulated evolution process, the phase separation of $\mathrm{Fe}_{65} \mathrm{Cu}_{35}$ alloy experiences a transformation from a dispersed structure into a triple-layer core-shell microstructure. $\mathrm{Fe}_{50} \mathrm{Cu}_{50}$ alloy follows the structural evolution route of "bicontinuous phase $\rightarrow$ quadruple-layer core-shell $\rightarrow$ triple-layer core-shell". Similarly, $\mathrm{Fe}_{35} \mathrm{Cu}_{65}$ alloy derives its final double-layer core-shell microstructure from an initial dispersed morphology.

(3) At the beginning of phase separation, the evolution of structural morphology is controlled by the reduction of surface free energy and bulk free energy. The effect of surface segregation results in the location of $\mathrm{Cu}$-rich phase at droplet surface and induces the appearance of concentration fluctuation. Later on, the interface free energy becomes the dominant factor. The Marangoni convection caused by the temperature gradient broadens the concentration fluctuation, makes it spread toward the droplet center and controls the final evolution of internal microstructures.

The authors are grateful to Luo Bingchi and Chang Jian for helpful discussions.

1 Carlberg T, Fredriksson H. The inflence of microgravity on the solidification of Zn-Bi immiscible alloys. Metall Trans, 1980, 12: $669-675$

2 Wang C P, Liu X J, Ohnuma I, et al. Formation of immiscible alloy powders with egg-type microstructure. Science, 2002, 297: $990-993$

3 Tegze G, Pusztai T, Gránásy L. Phase field simulation of liquid phase separation with fluid flow. Mater Sci Eng A, 2005, 413-414: $418-422$

4 Lu X Y, Cao C D, Wei B. Microstructure evolution of undercooled iron-copper hypoperitectic alloy. Mat Sci Eng A, 2001, 313: $198-206$

5 Tanaka H. Interplay between wetting and phase separation in binary fluid mixtures: Roles of hydrodynamics. J Phys: Condens Matter, 2001, 13: 4637-4674

6 Huo Y L, Zhang H D, Yang Y L. The morphology and dynamics of the viscoelastic microphase separation of diblock copolymers. Macromolecules, 2003, 36: 5383-5391

7 Liu X R, Lu X Y, Wei B B. Rapid monotectic solidification under free fall condition. Sci China Ser E-Eng Mater Sci, 2004, 47(4): 409-420

8 Hohenberg P C, Halperin B I. Theory of dynamic critical phenomena. Rev Mod Phys, 1977, 49: 435-479

9 Nakagana Y. Liquid immiscibility in copper-iron and copper-cobalt systems in the surpercooled state. Acta Metall, 1958, 6(11): $704-710$

10 Lu X Y, Cao C D, Wei B. Metastable phase separation of $\mathrm{Fe}_{50} \mathrm{Cu}_{50}$ hypoperitectic alloy under space simulation conditions. Acta Metall Sinica, 1999, 12(2): 198-204

11 Vladimirova N, Malagoli A, Mauri R. Two-dimensional model of phase segregation in liquid binary mixtures. Phys Rev E, 1999, 60: 6968-6977

12 Feng D, Jin G J. Condensed Matter Physics(I)(in Chinese). Beijing: Higher Education Press, 2003. 610 - 614

13 Mautits N M, Zvelindovsky A V, Sevink G J, et al. Hydrodynamic effects in three-dimensional microphase separation of block copolymers: Dynamic mean-field density functional approach. J Chem Phys, 1998, 108: 9150 - 9154

14 Mauri R, Shinnar R, Triantafyllou G. Spinodal decomposition in binary mixtures. Phys Rev E, 1996, 53: 2613-2623

15 Chen H, Chakrabarti A. Surface-directed spinodal decomposition: Hydrodynamic effects. Phys Rev E, 1997, 55: $5680-5688$ 